Manuscript prepared for

Review of Scientific Instruments

\title{
Filtered cathodic arc deposition with ion-species-selective bias
}

\author{
André Anders \\ Lawrence Berkeley National Laboratory, 1 Cyclotron Road, Berkeley, California 94720
}

Nitisak Pasaja

Lawrence Berkeley National Laboratory, 1 Cyclotron Road, Berkeley, California 94720, now at: Department of Physics, Faculty of Science, Chiang Mai University, Chiang Mai 50202, Thailand.

\section{Sakon Sansongsiri}

Lawrence Berkeley National Laboratory, 1 Cyclotron Road, Berkeley, California 94720, now at: Department of Physics, Faculty of Science, Chiang Mai University, Chiang Mai 50202, Thailand.

\section{Sunnie H.N. Lim}

Lawrence Berkeley National Laboratory, 1 Cyclotron Road, Berkeley, California 94720, now at: School of Applied Sciences (Applied Physics), RMIT University, Melbourne, Victoria 3001, Australia

Manuscript October5, 2006

Corresponding Author:

\author{
André Anders \\ Lawrence Berkeley National Laboratory \\ 1 Cyclotron Road, Berkeley, California 94720 \\ Tel. 510-486-6745 \\ Fax 510-486-4374 \\ aanders@lbl.gov
}

N. Pasaja, S. Sansongsiri, and S.H.N Lim acknowledge support by the Thai Commission on Higher Education Staff Development, the Royal Golden Jubilee Fellowship of Thailand, and the American Australian Association for the 2004 ANZ Fellowship, respectively. This work was supported by the U.S. Department of Energy under Contract No. DE-AC02-05CH11231. 


\title{
Filtered cathodic arc deposition with ion-species-selective bias
}

\author{
André Anders \\ Lawrence Berkeley National Laboratory, 1 Cyclotron Road, Berkeley, California 94720 \\ Nitisak Pasaja \\ Lawrence Berkeley National Laboratory, 1 Cyclotron Road, Berkeley, California 94720, \\ now at: Department of Physics, Faculty of Science, Chiang Mai University, Chiang Mai \\ 50202, Thailand.
}

\section{Sakon Sansongsiri}

Lawrence Berkeley National Laboratory, 1 Cyclotron Road, Berkeley, California 94720, now at: Department of Physics, Faculty of Science, Chiang Mai University, Chiang Mai 50202, Thailand.

\section{Sunnie H.N. Lim}

Lawrence Berkeley National Laboratory, 1 Cyclotron Road, Berkeley, California 94720, now at: School of Applied Sciences (Applied Physics), RMIT University, Melbourne, Victoria 3001, Australia

\begin{abstract}
A dual-cathode arc plasma source was combined with a computer-controlled bias amplifier such as to synchronize substrate bias with the pulsed production of plasma. In this way, bias can be applied in a material-selective way. The principle has been applied to the synthesis metal-doped diamond-like carbon films, where the bias was applied and adjusted when the carbon plasma was condensing, and the substrate was at ground when the metal was incorporated. In doing so, excessive sputtering by too-energetic metal ions can be avoided while the $\mathrm{sp}^{3} / \mathrm{sp}^{2}$ ratio can be adjusted. It is shown that the resistivity of the film can be tuned by this species-selective bias. The principle can be extended to multiple-material plasma sources and complex materials.
\end{abstract}




\section{INTRODUCTION: BIAS EFFECTS ON COATINGS}

Substrate bias is a well-known powerful tool to control the energy of ions in plasma-based thin film growth. A space charge sheath forms between the substrate surface and the plasma bulk. Typically, the substrate bias is negative such that positive ions are extracted from the sheath-plasma edge and accelerated before they impact the substrate surface ${ }^{1}$. Provided the sheath is much thinner than the mean free path between collisions, which is often the case, ions gain the kinetic energy

$$
\Delta E_{\text {kin }}=Q e \Delta V_{\text {sheath }}
$$

when traveling through the sheath; here $Q$ is the ion charge state number, $e$ is the elementary charge, and $\Delta V_{\text {sheath }}$ is the voltage drop between plasma sheath edge and the substrate surface. ${ }^{1}$ By controlling the energy of ions, film properties such as density and stress are affected and can be tuned and optimized for the desired application. ${ }^{2}$ In the case of crystalline films, preferred orientation and texture can be influenced, too. ${ }^{3}$

Of particular interests to some coating processes are highly ionized plasmas because bias techniques are very effective: bias acts only on ions but not on the neutral atoms. Examples of highly ionized plasmas are cathodic $\operatorname{arc}^{1}$ and HIPIMS (high power impulse magnetron sputtering) plasmas. ${ }^{4,5}$

In the literature, various forms of bias are known such as direct current (DC) bias, pulsed DC bias, radio frequency (RF) bias, etc. The bias acts on all ion species that enter the sheath from the plasma sheath edge. This, however, can be undesirable when ions of very different masses are involved: their sputter yields are very different, even when their final energy at arrival on the substrate surface is the same. The issue can be aggravated when the heavier ion has a higher charge state. In this case, the energy is enhanced 
proportional to the charge state, cf. Equ.(1), and therefore the sputter yield is much higher by the combination of greater mass and higher energy. An excessive sputter yield can be detrimental to making the desired coating composed of light and heavy atoms. Therefore, it is desirable to tune the bias in such a way as to adjust the value according to the species that are arriving. In this contribution, we will give further motivation why such species-selective bias is desirable by considering metal-doped carbon coatings, and we introduce a variation of a multiple-cathode coating system that allowed us to realize species-selective bias.

\section{DUAL CATHODIC ARC SYSTEM WITH SPECIES-SELECTIVE BIAS}

Let us consider the example of the synthesis of metal-doped tetrahedral amorphous carbon films. Such films, usually designated as ta-C:Me, can be made by filtered cathodic arc deposition. It is well established that the carbon ions should have an energy of about $100-120 \mathrm{eV}$ in order to produce a material rich in the tetrahedral (diamond) or $\mathrm{sp}^{3}$ bonding. ${ }^{6-9}$ Since the "natural" kinetic energy of carbon ions is only about $20 \mathrm{eV},{ }^{10}$ negative bias of typically about $100 \mathrm{~V}$ is applied to produce the most diamond-like material. If metal ions are produced by a cathodic arc, their most likely charge state is typically 2 or 3 (Table 1 ) and their most likely "natural" energy usually exceeds $50 \mathrm{eV}$ (Table 1). If the bias acts on the metal ions, their total kinetic energy can easily approach the range $300-500 \mathrm{eV}$, which is known to cause severe sputtering.

Moreover, the energetic impact will promote relaxation of $\mathrm{sp}^{3}$ bonds to $\mathrm{sp}^{2}$ bonds, thereby reducing the "diamondlikeness" of the material. 
To address the issue of excessive metal ion energy while having optimized carbon energy, a filtered dual-cathode cathodic arc system was developed that has speciesselective bias. On cathode was carbon, the other metal, and both were placed in close vicinity inside a common anode body (Fig. 1). The source is designed for pulsed operation, similar to our single-cathode "miniguns". ${ }^{11}$ Compared to the original miniguns, improved cooling allowed us to operate the dual-cathode source with pulses up to about $2 \mathrm{kA}$ with duration of typically $1 \mathrm{~ms}$, at a repetition rate of up to 10 pulses per second. The improved duty cycle is still low ( $1 \%$ or less) and the average current and power are about $10 \mathrm{~A}$ and $200 \mathrm{~W}$, respectively. The arc pulses were initiated simply by applying the open-circuit voltage (typically $600 \mathrm{~V}$ ) to the selected cathode; a hot spot developed at the edge to the insulator that separates the powered cathode from the anode body. Since no trigger electrode is required, we named this "triggerless” triggering. ${ }^{12}$

The dual-cathode plasma source was used to inject streaming cathodic arc plasma into a plasma filter, such as the open $90^{\circ}$ filter, ${ }^{13}$ to remove unwanted macroparticles. Because the injection points of plasma from the two cathodes are slightly different, the plasmas paths inside the filter and at the filter are not exactly the same. If deposition was made close to the exit of the filter, and the substrate was not moved, one would get two center regions of coatings that are offset with respect to each other, each center corresponding to one cathode material. This offset could be either utilized, if a combinatorial approach to materials research is desired, or one could minimize it by using large distances from the filter exit (more than $10 \mathrm{~cm}$ ) and substrate motion.

The key feature of this dual-cathode pulsed source is that the plasma generation can be selectively synchronized with the substrate bias. Specifically, bias pulses can be 
applied when carbon ions arrive at the substrate, such as to optimize the $\mathrm{sp}^{3}$ content of film, and reduced or omitted when metal plasma arrives at the substrate.

\section{EXPERIMENTS FOR ta-C:Mo FILMS}

In our setup, the initiation of arc pulses at a specific arc cathode and the timing of bias pulses were synchronized via a National Instruments ${ }^{\circledR}$ virtual instrument with LabView $^{\circledR}$ software (Fig. 2). This allowed us to write "recipes" for the film composition and structure in which the number of arc pulses, their sequence, and the amplitude of bias can be freely adjusted. In particular, the system allowed us to produce ta-C:Mo where the carbon ions "saw" bias whereas the molybdenum ions arrived at an unbiased (grounded) substrate. In this sense, “species-selective” biasing has been realized.

Fig. 3 shows a result of the ta-C:Mo experiments. Here, the amplitude of the bias for carbon plasma was varied with the goal to tune the $\mathrm{sp}^{3} / \mathrm{sp}^{2}$ ratio and thereby adjust the related optical and electrical properties, specially the resistivity, while keeping the metal content at a constant level. The every $21^{\text {st }}$ pulse was a metal pulse, and the metal was deposited with the substrate at ground. This is an example for the flexibility of the deposition approach using species-selective bias.

Film synthesis using two filtered pulsed arc sources of the "minigun” style has been demonstrated previously. A diamond-like carbon (DLC) / TiC multilayers as well as monolithic $\mathrm{Si}_{\mathrm{x}} \mathrm{Al}_{\mathrm{y}} \mathrm{O}$ (mullite) were deposited using $\mathrm{Ti}$ and $\mathrm{C}$, and $\mathrm{Si}$ and $\mathrm{Al}$ cathodes, respectively. ${ }^{14}$ Each of the two sources had its own filter, aiming the filtered plasma streams to a common substrate location. The same system was used to produce $\mathrm{WC}_{\mathrm{x}}$ with various metal concentrations. ${ }^{15}$ 
A dual-cathode arc source, similar to the one presented here but with a trigger electrode placed at the center of each cathode, was employed by Davies and coworkers. ${ }^{16}$ They used their system to produce $\mathrm{Ti}_{1-\mathrm{x}} \mathrm{V}_{\mathrm{x}} \mathrm{N}$ with controlled composition, finding that $x=0.23$ produces the highest indentation hardness and hence the highest yield stress. Although these approaches to materials synthesis are similar, the various ion species where not accelerated in a bias-selective manner.

\section{GENERALIZATION}

\section{A. Ion etching and implantation versus deposition}

In principle, the approach can also be used to use alternate phases of deposition of one species (relatively low bias) and etching / subplantation / implantation of another species (at relatively high bias). This hybrid principle was first proposed by Brown and

coworkers using a single-material cathodic arc plasma source. ${ }^{17,18}$ Using a dual-cathode source, the possibilities of metal plasma immersion ion implantation and deposition (MePIIID ${ }^{1}$ ) are extended by choosing arbitrary combinations (“recipes”) of materials and energy by selectively energizing the arc plasma pulses in desired phases with bias pulses.

\section{B. Multiple material plasma sources}

It is straightforward to increase the number of cathodes to three or four, thereby increasing the versatility and possibilities even further. As already indicated, the background gas can be yet another source of material: the cathodic arc plasma sources can be used in reactive mode, producing compound films but utilizing the presence of reactive gases in the chamber (oxygen for oxides, ${ }^{19}$ nitrogen for nitrides, ${ }^{16}$ etc.). With 
these extensions, it is anticipated that a great variety of complex systems can be produced. For example, it might be possible to use a system with three cathodes (Y, Ba, $\mathrm{Cu}$ ), operating in an oxygen background gas, to synthesize YBCO high- $T_{\mathrm{c}}$ superconducting films. Other examples are transparent magnetic semiconductors ${ }^{20}$ such as $\mathrm{ZnO}: \mathrm{Cr}$, or transparent electronics ${ }^{21}$ based on $\mathrm{ZnO}: \mathrm{M}$, where " $\mathrm{M}$ ” is a dopant that produces $n$-type or $p$-type conductivity. Other complex systems are ternary oxides that show colossal magnetoresistance ${ }^{22}$ such as $\mathrm{Nd}_{0.7} \mathrm{Sr}_{0.3} \mathrm{MnO}_{3}$, or multiferroics ${ }^{23}$ such as $\mathrm{BiFeO}_{3}, \mathrm{Bi}_{2} \mathrm{FeCrO}_{6}, \mathrm{BiCrO}_{3}, \mathrm{LaTiO}_{3}$, and $\mathrm{SrTiO}_{3}$.

The cathodes of the multiple-cathode plasma source are usually operated sequentially, to synthesize films with mixed material content or to deposit multilayers, as determined by the recipe of the process. However, we have also demonstrated simultaneous cathode operation using either a single power supply with a low-ohm distributing circuit, or by using two individual power supplies, each dedicated to a single cathode. Such simultaneous operation may be beneficial when the components need to react with each other rather than with the residual gas of the background vacuum.

\section{SUMMARY}

In summary, a compact pulsed cathodic arc plasma source has been developed with two "triggerless" cathodes in a common anode body, allowing materials-selective bias by synchronizing the bias amplitude with the presence of the plasma of a specified material. The principle has been illustrated using carbon and molybdenum as the two cathode materials. In the example, the bias voltage has been systematically adjusted for carbon only such as to obtain a certain electrical resistivity of the growing film, while the 
metal deposition was not affect. The approach using arc plasma sources with multiple cathodes and selective application of bias opens the possibilities of making a great variety of films and multilayers of mixed and complex composition.

\section{ACKNOWLEDGEMENTS}

N. Pasaja, S. Sansongsiri, and S.H.N Lim acknowledge support by the Thai Commission on Higher Education Staff Development, the Royal Golden Jubilee Fellowship of Thailand, and the American Australian Association for the 2004 ANZ Fellowship, respectively. The authors thank Joel Teague, Joe Wallig, Michael Dickinson, and Tom McVeigh for technical support. This work was supported by the U.S. Department of Energy under Contract No. DE-AC02-05CH11231. 


\section{References}

1 A. Anders, Surf. Coat. Technol. 93, 157 (1997).

2 M. M. M. Bilek, R. N. Tarrant, D. R. McKenzie, S. H. N. Lim, and D. G. McCulloch, IEEE Trans. Plasma Sci. 31, 939 (2003).

3 B. K. Gan, M. M. M. Bilek, D. R. McKenzie, S. Yang, D. A. Tompsett, M. B. Taylor, and D. G. McCulloch, J. Phys. Condensed Matter 16, 1751 (2004).

4 A. P. Ehiasarian, R. New, W.-D. Münz, L. Hultman, U. Helmersson, and V. Kouznetsov, Vacuum 65, 147 (2002).

5 J. Bohlmark, J. Alami, C. Christou, A. Ehiasarian, and U. Helmersson, J. Vac. Sci. Technol. A 23, 18 (2005).

6 P. J. Fallon, V. S. Veerasamy, C. A. Davis, J. Robertson, G. A. J. Amaratunga, W. I. Milne, and J. Koskinen, Phys. Rev. B 48, 4777 (1993).

$7 \quad$ Y. Lifshitz, G. D. Lempert, S. Rotter, I. Avigal, C. Uzan-Saguy, R. Kalish, D. Marton, and J. W. Rabalais, Diamond Rel. Mat. 3, 542 (1994).

8 E. Grossman, G. D. Lempert, J. Kulik, D. Marton, J. W. Rabalais, and Y. Lifshitz, Appl. Phys. Lett. 68, 1214 (1996).

9 M. Hakovirta, J. Salo, R. Lappalainen, and A. Anttila, Phys. Lett. A 205, 287 (1995).

10 A. Anders and G. Y. Yushkov, J. Appl. Phys. 91, 4824 (2002).

11 R. A. MacGill, M. R. Dickinson, A. Anders, O. R. Monteiro, and I. G. Brown, Rev. Sci. Instrum. 69, 801 (1998).

12 A. Anders, I. G. Brown, R. A. MacGill, and M. R. Dickinson, J. Phys. D: Appl. Phys. 31, 584 (1998). 
13 A. Anders, Surf. Coat. Technol. 120-121, 319 (1999).

14 O. R. Monteiro, Z. Wang, P. Y. Hou, and I. G. Brown, Nucl. Instrum. Meth. Phys. Res. B 127/128, 821 (1997).

15 O. R. Monteiro, M.-P. Delplancke-Ogletree, R. Y. Lo, R. Winand, and I. G. Brown, Surf. Coat. Technol. 94-95, 220 (1997).

16 K. E. Davies, B. K. Gan, D. R. McKenzie, M. M. M. Bilek, M. B. Taylor, D. G. McCulloch, and B. A. Latella, J. Phys. Condensed Matter 16, 7947 (2004).

17 I. G. Brown, X. Godechot, and K. M. Yu, Appl. Phys. Lett. 58, 1392 (1991).

18 I. G. Brown, A. Anders, S. Anders, M. R. Dickinson, I. C. Ivanov, R. A. MacGill, X. Y. Yao, and K.-M. Yu, Nucl. Instrum. Meth. Phys. Res. B 80/81, 1281 (1993).

19 O. R. Monteiro, Z. Wang, and I. G. Brown, J. Mater. Res. 12, 2401 (1997).

20 I. Satoh and T. Kobayashi, Appl. Surf. Sci. 216, 603 (2003).

21 J. G. Lu, L. P. Zhu, Z. Z. Ye, F. Zhuge, Y. J. Zeng, B. H. Zhao, and D. W. Ma, Applied Surface Science 245, 109 (2005).

22 V. N. Smolyaninova, X. C. Xie, F. C. Zhang, M. Rajeswari, R. L. Greene, and S. D. Sarma, Phys. Rev. B 62, 3010 (2000).

23 P. Baettig, C. Ederer, and N. A. Spaldin, Phys. Rev. B 72, 214105 (2005).

24 A. Anders, Phys. Rev. E 55, 969 (1997). 


\section{Figure Captions}

Fig. 1 Photograph of a cathodic arc plasma source with two cathodes in a common, water-cooled anode body.

Fig. 2 Schematic of the system using a dual-cathode plasma source and selective, synchronized initiation of arc pulses and bias pulses. The personal computer (PC) is

equipped with a National Instruments ${ }^{\circledR}$ virtual instrument with LabView ${ }^{\circledR}$ software that allowed us to write "recipes” for the synchronization of bias and arc pulses. (The line in the center of the chamber indicates a macroparticle "firewall," i.e. a physical blockage for particle emanating from the arc source and filer region; the substrate may be movable, which is not further shown here).

Fig. 3 Resistivity of ta-C:Mo films with the pulsed bias acting only on the carbon ions (pulsed bias: $2 \mu$ s on and $6 \mu$ s off, for the duration of the presence of carbon plasma). The substrate was at ground when molybdenum plasma arrived. The molybdenum to carbon arc pulse ratio was kept constant at 1:20. 


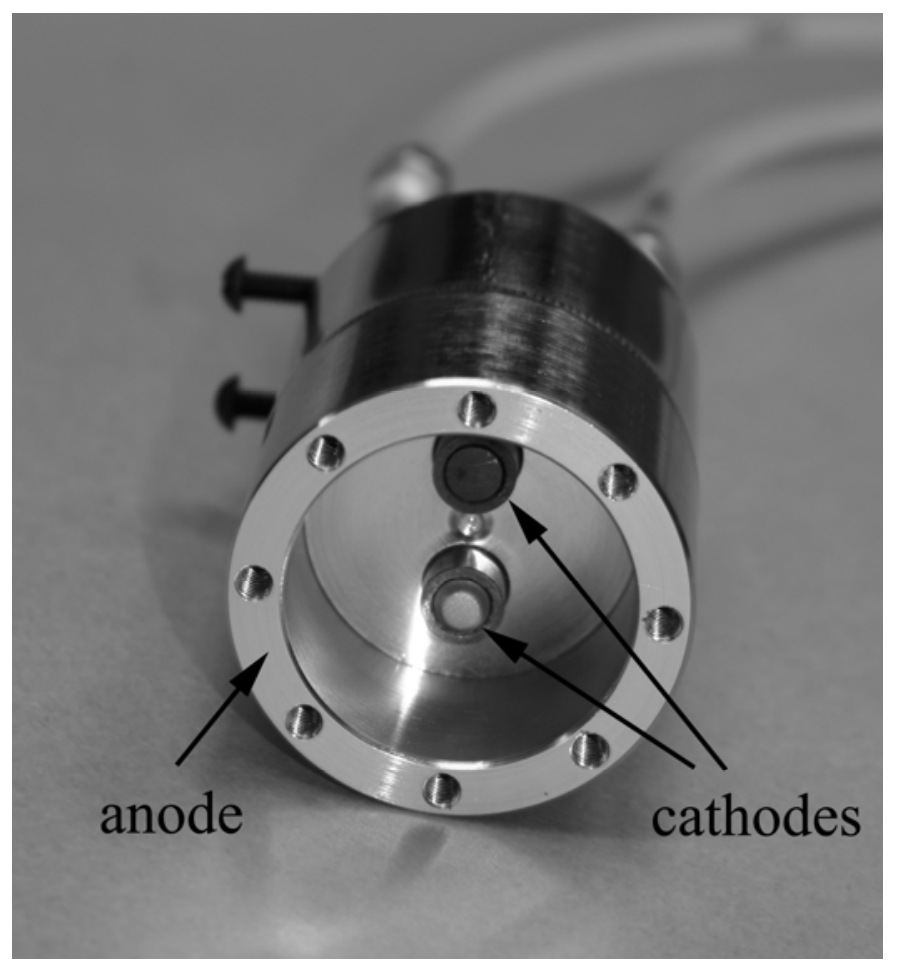

Fig. 1 


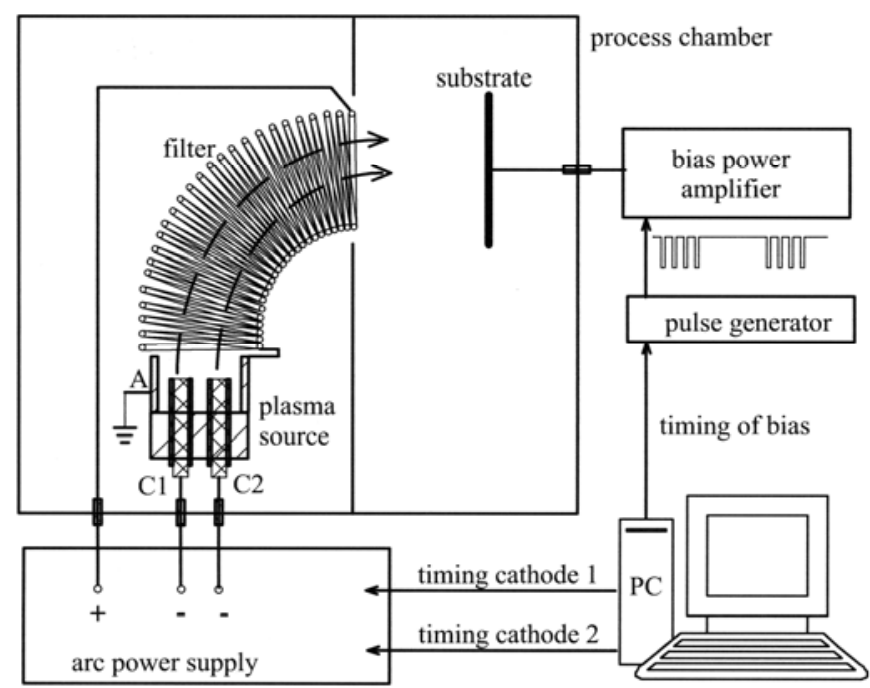

Fig. 2 


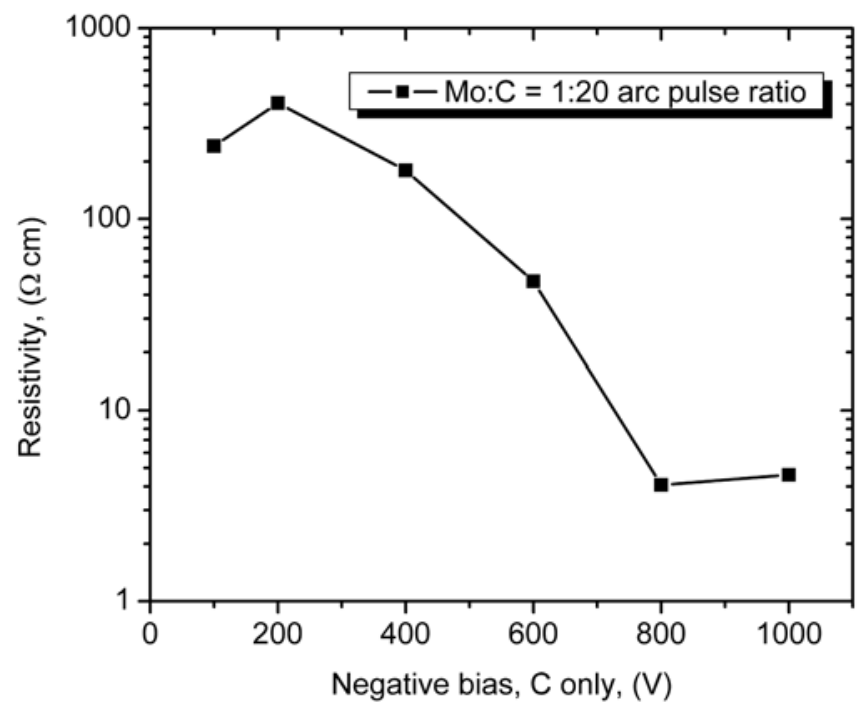

Fig. 3 


\section{Table 1}

Average ion charge state, $\bar{Q}$, particle charge state fractions, and the most likely "natural" kinetic energy of ions of cathodic arc plasmas. The selection was made for some of the relevant elements; comprehensive data an be found in the literature, e.g. ${ }^{10,24}$.

\begin{tabular}{ccccccccc}
\hline Material & $\bar{Q}$ & $f_{1}$ & $f_{2}$ & $f_{3}$ & $f_{4}$ & $f_{5}$ & $f_{6}$ & $E_{\text {kin }}$ \\
& & & & & & & & $(\mathrm{eV})$ \\
\hline $\mathrm{C}$ & 1.00 & 100 & & & & & 19 \\
$\mathrm{Ti}$ & 2.03 & 11 & 75 & 14 & & & & 59 \\
$\mathrm{Cr}$ & 2.09 & 10 & 68 & 21 & 1 & & & 72 \\
$\mathrm{Mo}$ & 3.06 & 2 & 21 & 49 & 25 & 3 & & 149 \\
$\mathrm{~W}$ & 3.07 & 2 & 23 & 43 & 26 & 5 & 1 & 117 \\
\hline
\end{tabular}

